Rolinson, G. N. \& Lumb, M. (1953). J. gen. Microbiol. 8, 265-272.

\title{
The Effect of Aeration on the Utilization of Respiratory Substrates by Penicillium chrysogenum in Sub- merged Culture
}

\author{
By G. N. ROLINSON AND M. LUMB \\ Research Department, Bacteriology Division, Boots Pure \\ Drug Co. Ltd., Nottingham
}

SUMMARY: At high aeration rates in submerged culture, lard oil (mainly glycerides of oleic and stearic acids) may be utilized by Penicillium chrysogenum as a source of carbon in preference to carbohydrate. At relatively low aeration rates, carbohydrate serves as the principal carbon source.

Enzymes involved in the utilization of lard oil appear to be synthesized by the growing mycelium, this synthesis being favoured by conditions of high aeration.

In an earlier paper (Rolinson, 1952) it was shown that the degree of aeration in spore-inoculated penicillin fermentations affects not only the rate of respiration but also the kind of metabolism carried out. In shaken flask fermentations, the R.Q. of the mycelium was $c .1 \cdot 0$ throughout the fermentation, whereas in fermentations at very high aeration rates the R.Q. of the mycelium was c. $1 \cdot 0$ only during the first $30 \mathrm{hr}$., then fell to values as low as $0 \cdot 6$ as the fermentation proceeded. The R.Q. values for the two types of mycelium were little affected by the immediate degree of aeration. Thus mycelium grown at low aeration rates continued to respire with an R.Q. of $c .1 \cdot 0$, even though an excess of dissolved oxygen was present, and conversely the low R.Q. of the mycelium from the highly aerated fermentations was still evident when respiration was limited by the concentration of dissolved oxygen. The differences in metabolism, as shown by the different R.Q. values, therefore appeared to indicate the presence of different enzyme systems in the mycelia, having presumably been developed as a result of the differences in concentration of dissolved oxygen during growth.

The metabolism of mycelium of Penicillium chrysogenum has been studied further, and the present paper concerns the respiratory substrates utilized by the two types of mycelium and which are responsible for the differences in the R.Q. observed previously.

\section{METHODS}

\section{Fermentation methods}

Laboratory fermenters of the type described by Lumb \& Fawcett (1951) using 3 l. of medium were operated at different rates of stirring and air flow. Shaken flask fermentations were carried out with $75 \mathrm{ml}$. lots of medium in $250 \mathrm{ml}$. conical Pyrex or Hysil flasks on a rotary shaker (speed 220 r.p.m.; throw of 1.5 in.). All fermentations were carried out at $24-26^{\circ}$.

The medium used in all the experiments contained $(\% \mathrm{w} / \mathrm{v})$ : corn steep liquor (solids basis), 1.5 ; lactose, 3.0 ; glucose, $0.5 ; \mathrm{NaNO}_{3}, 0.3 ; \mathrm{KH}_{2} \mathrm{PO}_{4}$, 
$0 \cdot 05 ; \mathrm{Na}_{2} \mathrm{SO}_{4}, 0 \cdot 1 ; \mathrm{pH}$ value adjusted to $5 \cdot 8$. Phenylacetic acid was added to the stirred and aerated fermentations in additions of $0.05 \%(\mathrm{w} / \mathrm{v})$ every $12 \mathrm{hr}$., starting at the 24th $\mathrm{hr}$. In the shaken flask fermentations, a concentration of $0 \cdot 25 \%(\mathrm{w} / \mathrm{v})$ phenylacetic acid was added to the medium initially. Lard oil containing $2.5 \%(\mathrm{v} / \mathrm{v})$ cetyl alcohol was used as an antifoam agent as required. The lard oil, which consists mainly of glycerides of oleic and stearic acids, was obtained from Messrs Brown and Deighton, Preston, Lancashire. All the fermentations were inoculated with spore suspensions prepared from lyophilized master cultures of $\boldsymbol{P}$. chrysogenum W 48-701.

\section{Analytical methods}

Glucose and lactose were estimated by determining the reducing power of the sample before and after hydrolysis. The difference between the two values was taken to be equivalent to half the quantity of lactose present in the sample from which the separate quantities of glucose and lactose were calculated. The sample was hydrolysed by boiling with $\mathrm{N}-\mathrm{HCl}$ for $45 \mathrm{~min}$. Reducing power was estimated by the following modification of the Folin-Wu (1920) method. The sample was filtered through kieselguhr and diluted so that $3 \mathrm{ml}$. contained less


with 2 ml. of Folin's alkaline cupric solution in a Folin tube. The tube was then placed in cold water for 1 min. After boiling again for 2 min. with $2 \mathrm{ml}$. of Folin's phosphomolybdate solution the tube was again placed in cold water for $1 \mathrm{~min}$. before being made up to the $25 \mathrm{ml}$. mark with distilled water. The contents of the tube were then mixed and read immediately in a Spekker absorptiometer using yellow filters (Hilger 606). Reducing power was read from a calibration curve prepared with standard solutions of glucose. During boiling with the phosphomolybdate solution, small glass funnels were placed in the necks of the Folin tubes to prevent reoxidation by the atmosphere.

Total Kjeldahl nitrogen was determined by the conventional procedure using a Markham distillation apparatus; amino-nitrogen was determined by Sørensen's formol titration method; mycelial dry weight was determined as described by Rolinson (1952). Conventional Warburg manometric techniques were used as described by Umbreit, Burris \& Stauffer (1945).

\section{RESULTS}

\section{Utilization of carbohydrates}

The usage of glucose and lactose in fermentations at high aeration rates and in fermentations in shaken flasks (relatively low aeration) is shown in Figs. 1 and 2. It will be seen that in the shaken flask fermentations glucose and lactose were both used rapidly and completely, the residual carbohydrate at the end of the fermentation being usually negligible. In the highly aerated fermentations however, although the glucose was again completely used, lactose was only utilized rapidly during about the first $30 \mathrm{hr}$. After this time the rate of lactose utilization was slow, and at the end of fermentation usually as much as $60 \%$ of the initial lactose remained in the fermentation medium. 
It has been shown (Rolinson, 1952) that extremely high rates of solution of oxygen are obtained in these laboratory fermenters as compared with the rates in shaken flasks, and it therefore appeared from the results shown in Figs. 1 and 2 that aeration above the degree obtained in shaken flasks inversely affected the rate of lactose usage. Accordingly, fermentations were carried out in the



Fig. 1

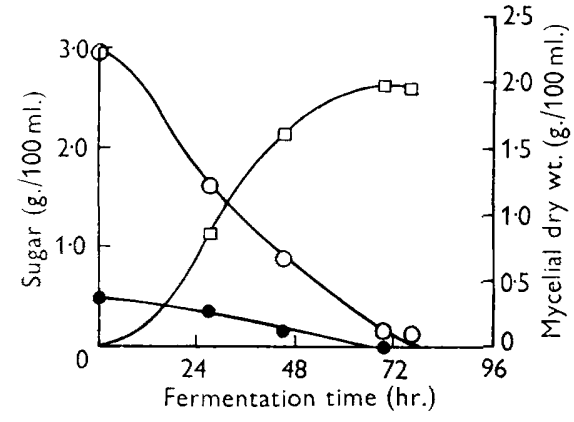

Fig. 2

Fig. 1. Growth and usage of lactose and glucose in a highly aerated fermentation. Stirring 1000 r.p.m., air flow 2 vol./vol./min. Mycelial dry wt., $\square-\square$; lactose, $\bigcirc-\bigcirc$; glucose, $0-0$.

Fig. 2. Growth and usage of lactose and glucose in a shaken flask fermentation. Mycelial dry wt., $\square-\square$; lactose, $\bigcirc-\bigcirc$; glucose,

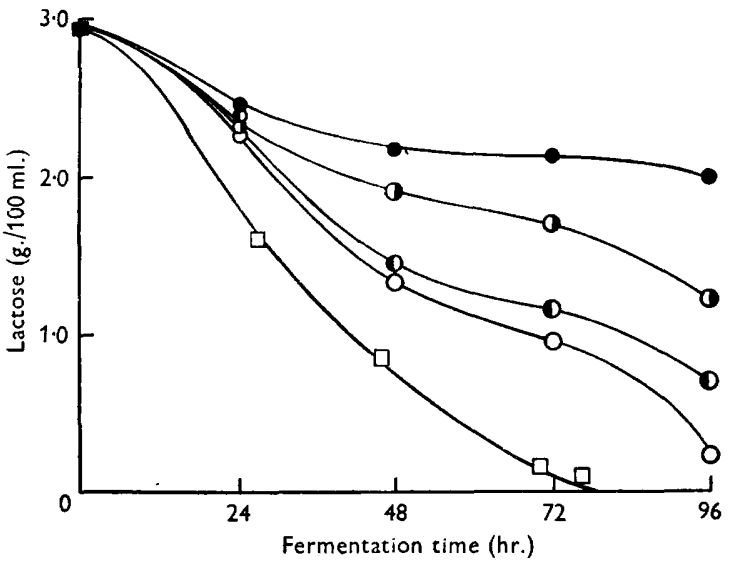

Fig. 3. Effect of rates of stirring and air flow on the usage of lactose in fermentations in laboratory fermenters. 1000 r.p.m. and 2 vol./vol./min.,

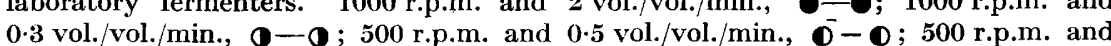
$0 \cdot 3 \mathrm{vol} . / \mathrm{vol} . \mathrm{min}$., $\mathrm{O}-\mathrm{O}$. Shaken flask fermentation, $\square-\square$.

laboratory fermenters under different conditions of aeration, obtained by varying the rates of stirring and air flow (see Fig. 3), which resulted in progressively altered rates of solution of oxygen in the fermentation medium as determined by the method of Wise (1951). The usage of lactose under different degrees of aeration thus obtained is shown in Fig. 3. It will be seen that increased aeration resulted in diminished rates of lactose usage, the lowest 
rate occurring with the highest degree of aeration. Lactose usage was also depressed when the degree of aeration was below a certain value. By increasing the volumes of medium used in the shaken flasks the rate of solution of oxygen could be so decreased that both lactose usage and the amount of mycelium formed were markedly diminished. The incomplete usage of lactose under conditions of high aeration, however, was not accompanied by a corresponding diminution in the amount of mycelial growth. This suggested that, under con-

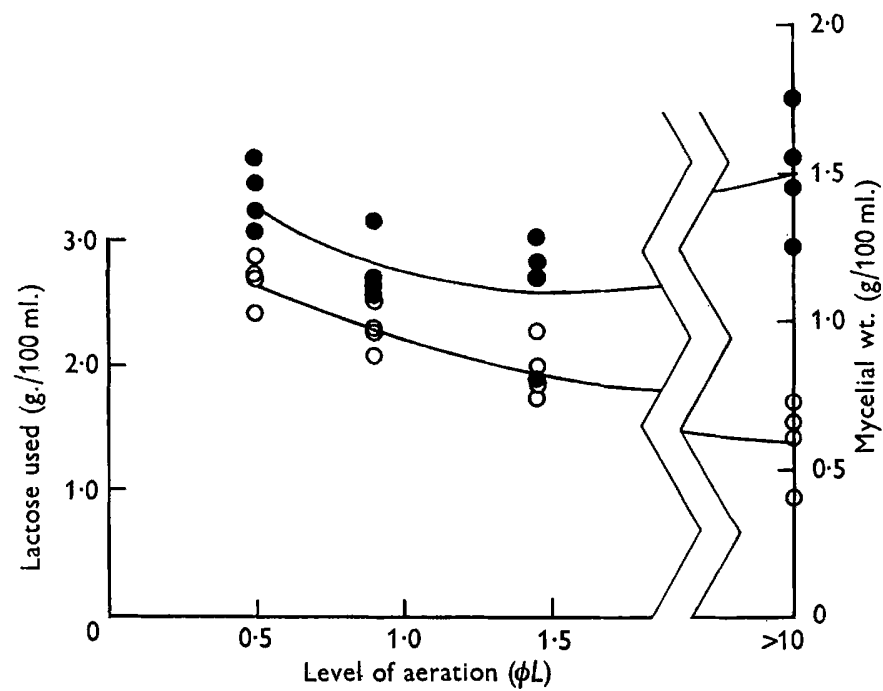

Fig. 4. Usage of lactose and the formation of mycelium after $96 \mathrm{hr}$. incubation at different degrees of aeration. Lactose used, $\mathrm{O}-\mathrm{O}$; mycelial dry weight, - - . Level of aeration $(\phi L)$ determined by the polarographic gassing-out method of Wise (1951) using a solution of $\mathrm{KCl}$ in the fermentation vessel. The rates of stirring and air flow used were; $1000 \mathrm{r} . \mathrm{p} . \mathrm{m}$. and $2 \mathrm{vol} . / \mathrm{vol} . / \mathrm{min} ., 1000 \mathrm{r.p.m}$. and $0.3 \mathrm{vol} . / \mathrm{vol} . / \mathrm{min} ., 500 \mathrm{r.p.m}$. and $0.5 \mathrm{vol} . / \mathrm{vol} . / \mathrm{min}$. and $500 \mathrm{r} . \mathrm{p} . \mathrm{m}$. and $0.3 \mathrm{vol} . / \mathrm{vol} . / \mathrm{min}$. giving $\phi L$ values of $>10,1.4$, $0 \cdot 8$ and 0.5 respectively.

ditions of high aeration, some substrate was being utilized as an alternative to lactose. This alternative source of carbon did not appear to be used at all degrees of aeration. When mycelial growth and usage of lactose were plotted against the degree of aeration (Fig. 4) it was found that an increase of aeration from that obtained with 500 r.p.m. and $0.3 \mathrm{vol}$. air $/ \mathrm{vol}$. medium $/ \mathrm{min}$. to that with $1000 \mathrm{r} . \mathrm{p} . \mathrm{m}$. and $\mathbf{0 . 3} \mathrm{vol} . / \mathrm{vol} . / \mathrm{min}$. resulted in a lower mycelial weight, corresponding to the fall in amount of lactose used. Under such conditions there appeared to be no alternative substrate to compensate for the decrease in lactose consumption. At the maximum degree of aeration, however (1000r.p.m., $2 \mathrm{vol} . / \mathrm{vol} . / \mathrm{min}$.), the rate of lactose usage was still further diminished, although the growth was equal to that obtained with the lowest degree of aeration when all the available lactose was used. Only at the highest degree of aeration, therefore, was some alternative substrate being utilized efficiently enough to enable growth to be as massive as when carbohydrate was being used. The only substances present in the medium in sufficient 
quantity to provide the necessary carbon for the amount of growth made when lactose usage was very incomplete were the proteins and amino-acids of the corn-steep liquor and the lard oil added as a vehicle for the antifoam agent. The quantity of lard oil thus added frequently amounted to as much as $1 \%(\mathrm{v} / \mathrm{v})$.

\section{Utilization of nitrogenous constituents of the medium}

The usage of the organic nitrogenous constituents of the medium was examined, and it was found that their utilization was not significantly influenced by the degree of aeration. The latter substances therefore did not appear to be the alternative carbon source utilized under conditions of high aeration.

\section{Utilization of lard oil}

The total amount of lard oil added during two separate fermentations $($ c. $1.0 \%, \mathrm{v} / \mathrm{v})$ was recorded, and the residual fat extracted from the broth at the end of the fermentation by shaking $500 \mathrm{ml}$. volumes with two successive $100 \mathrm{ml}$. volumes of chloroform. The chloroform extracts were combined with chloroform washings of the interior of the fermenter, evaporated and weighed. The results are shown in Table 1. It will be seen from these results that only c. $5 \%$ of the total lard oil added was recovered at the end of the fermentation.

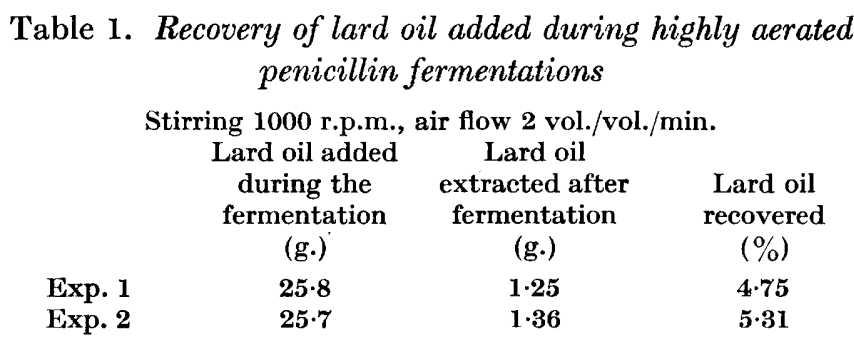

Evidence that lard oil could be rapidly oxidized was obtained from manometric experiments with washed mycelium.

Respiration of mycelium from highly aerated fermentations. Samples of fermentation brew were removed and the mycelium washed four times by centrifuging and suspending in a solution containing only the inorganic constituents of the medium (salts solution). The concentration of mycelium in these suspensions was similar to that in the fermentation at the time of sampling. The $\mathrm{pH}$ value of the salts solution was also adjusted to that of the fermentation at the time of sampling. Oxygen uptake was measured in Warburg manometers at $26^{\circ}$ rocked at 120 strokes $/ \mathrm{min}$. Each flask contained $\mathbf{1 . 0} \mathrm{ml}$. of mycelial suspension together with a further $\mathbf{1 . 0} \mathrm{ml}$. of the salts solution to prevent the mycelium from becoming too densely packed in the flask.

The respiration of washed $46 \mathrm{hr}$. mycelium from a high aeration rate fermentation, in the presence of various substrates, is shown in Fig. 5. The relatively high endogenous rate of respiration, which is typical of fungi, was not diminished by further washing. It will be seen from Fig. 5 that not only 
was lard oil oxidized rapidly but the rate of respiration with lard oil as substrate was higher than with glucose or complete fermentation medium.

Respiration of mycelium from shaken flask fermentations. Mycelium $69 \mathrm{hr}$. old from shaken flask fermentations (relatively low degree of aeration) was washed and suspended in salts solution as before and respiration measured in the presence of different substrates. It will be seen from Fig. 6 that although glucose was oxidized rapidly, the presence of lard oil did not result in any

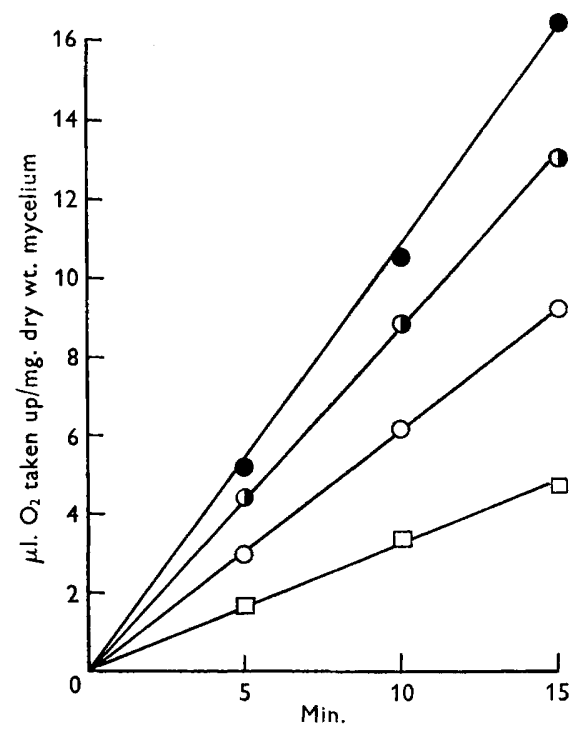

Fig. 5



Fig. 6

Fig. 5. Respiration of washed, $46 \mathrm{hr}$. mycelium of $P$. chrysogenum from a fermentation carried out in a laboratory fermenter stirred at 1000 r.p.m. with an air flow of $2 \mathrm{vol} . / \mathrm{vol} . / \mathrm{min}$. Substrates: $1 \%(\mathrm{v} / \mathrm{v})$ lard oil, - - ; complete sterile fermentation medium, - - ; $1 \%(\mathrm{w} / \mathrm{v})$ glucose, $\bigcirc-0$. Endogenous respiration, $\square-\square$.

Fig. 6. Respiration of washed $69 \mathrm{hr}$. mycelium of $P$. chrysogenum from a shaken flask fermentation. Substrates : $1 \%(v / v)$ lard oil, - $; 1 \%(w / v)$ glucose, $\bigcirc-0$. Endogenous respiration, $\square-\square$.

significant increase in respiration above the endogenous rate with mycelium from low aeration flasks. Thus if lard oil was metabolized by mycelium from shaken flasks it was certainly oxidized at a very much lower rate than by mycelium from the fermentations at high aeration rate. Since all the manometric experiments were carried out in flasks rocked at the same rate of 120 strokes/min., the difference in oxidation of lard oil by the two types of mycelium appeared to indicate a difference in enzyme content.

\section{DISCUSSION}

The effect of high aeration rates on lactose usage appears to be peculiar to fermentations inoculated with spores. In fermentations starting with a vegetative inoculum and with lard oil as vehicle for the antifoam agent, lactose was used rapidly and completely even at the highest degrees of aeration. There is, 
indeed, evidence that the usage of lactose is favoured by high aeration rates in fermentations started by inoculation with vegetative growth (Bartholomew, Karow, Sfat \& Wilhelm, 1950; Brown \& Peterson, 1950 a,b). In fermentations inoculated with vegetative growth, lard oil and related substances may also be metabolized as in spore-inoculated fermentations but evidently not in preference to carbohydrate.

The R.Q. values reported by Rolinson (1952) indicated a difference in metabolism between mycelium grown at high aeration rates and that grown at relatively low aeration rates. It is clear from the present results that one such difference lies in the relative ability of the two kinds of mycelium to oxidize lard oil. Only with the highest degree of aeration was lard oil utilized extensively and in preference to lactose. It is not clear whether high concentrations of dissolved oxygen directly inhibit the usage of lactose or whether the metabolism of lard oil involves some mechanism which is also involved in the utilization of lactose so that lard oil might diminish lactose usage by competition for this common mechanism. The results shown in Fig. 4 appear to indicate that increasing concentrations of dissolved oxygen do directly inhibit the utilization of lactose, since a limited increase in degree of aeration resulted in a fall in mycelial weight which corresponded to the fall in the amount of lactose used. Only when the aeration was increased to 1000 r.p.m. with air flow of $2 \mathrm{vol} . / \mathrm{vol} . / \mathrm{min}$. was some other substrate utilized efficiently as an alternative to lactose. Alternatively, the diminished uptake of lactose might nevertheless be due to competition with lard oil for some common mechanism, with the metabolism of lard oil serving in the synthesis of mycelium relatively inefficiently except under the highest degree of aeration.

Evidence has been presented (Rolinson, 1952) that differences in R.Q. values between mycelia from fermentations at different degrees of aeration represent a difference in enzyme content rather than a difference in response to the immediate condition of aeration. This is also borne out by the experiments described here. Mycelium from shaken flask fermentations showed no detectable oxidation of lard oil despite the excess of dissolved oxygen in the Warburg flasks, while these conditions permitted mycelium grown under conditions of high aeration to oxidize lard oil rapidly. These results suggest that enzymes involved in the metabolism of lard oil are synthesized during spore-inoculated penicillin fermentations and that the synthesis is favoured by conditions of high aeration.

The authors are indebted to the late Sir Jack Drummond, F.R.S., and Mr C. E. Coulthard for their interest in the work, to Mr R. Fawcett for assistance in carrying out the fermentations and Mr C. K. Mercer and Mr J. Ransom for technical assistance.

\section{REFERENCES}

Bartholomew, W. H., Karow, E. D., Sfat, M. R. \& Wilhelm, R. H. (1950). Effect of air flow and agitation rates upon fermentation of Penicillium chrysogenum and Streptomyces griseus. Industr. Engng. Chem. 42, 1810.

Brown, E. W. \& Peterson, W. H. (1950a). Factors affecting production of penicillin in semi-pilot plant equipment. Industr. Engng. Chem. 42, 1769. 
Brown, E. W. \& Peterson, W. H. (1950b). Penicillin fermentations in a Waldhoftype fermenter. Industr. Engng. Chem. 42, 1823.

Folin, O. \& Wu, H. (1920). A simplified and improved method for determination of sugar. J. biol. Chem. 41, 367.

Lumb, M. \& FAwCETT, R. (1951). Improvements in experimental fermenters. J. appl. Chem. 1, S 94.

Rolinson, G. N. (1952). Respiration of Penicillium chrysogenum in penicillin fermentations. J. gen. Microbiol. 6, 336.

Umbreit, W. W., Burris, R. H. \& Stauffer, J. F. (1945). Manometric techniques and related methods for the study of tissue metabolism. Minneapolis, Minn.: Burgess. Publ. Co.

WISE, W. S. (1951). The measurement of the aeration of culture media. J. gen. Microbiol. 5, 167.

(Received 19 August 1952) 